weight babies. There will be then also a need for an intravenous line. A short non-invasive ophthalmic procedure is changed into a lengthy and invasive anaesthesia technique.

Our previous experience with a nasal ketamine midazolam mixture in babies suggests that it is an attractive alternative for short ophthalmic procedures. A study is under way to use it for different procedures in babies and small children such as ophthalmic examinations, YAG laser treatment, and cryotherapy for ROP.

Nasal administration of drugs is not a new idea and has been used for some time. Pharmacokinetics of intranasal midazolam at a dose as low as $0.2 \mathrm{mg} / \mathrm{kg}$ showed that the hypnotic threshold of $100 \mathrm{ng} / \mathrm{ml}$ was obtained with 5-10 minutes and this correlated well with sedation. ${ }^{1}$ Pharmacokinetics of intranasal ketamine were studied by Russel and Aldrete. ${ }^{2}$ The dose was $4-6 \mathrm{mg} / \mathrm{kg}$ and the onset of action within 8-12 minutes. Compared with intramuscular or intravenous routes, intranasal administration is a noninvasive, pain-free method and has a fast onset of action. ${ }^{3-5}$ High bioavailability, rapid peak serum levels, and perhaps absorption through the cribriform plate result in a rapid onset of sedation. ${ }^{3}$

We would like to stress that this technique requires skilful supervision and adequate monitoring. We do not recommend it to be used by untrained staff in outpatient departments or outside the hospital. Low birthweight babies should recover in a high dependency area.

The absence of respiratory depression, arterial oxygen desaturation with satisfactory sedation suggests that this technique is safe and efficient for non-invasive ophthalmic procedures. A large series is required to evaluate potential side effects in this specific indication.

We have presented a novel technique of intranasal administration in a low weight baby requiring cryotherapy for ROP. The side effects were minimal and the operating conditions excellent.

1 Malinovsky JM, Le Normand Y, de Dieuleveult, Lepage JY, Cozian A. Midazolam pharmacokinetics in children after intranasal administration. Anesthesiology 1990; 73 (suppl):

2 Aldrete JA, Roman de Jesus JC, Russel LJ. Intranasal ketamine as induction adjunct in children: preliminary report. Anesthesiology 1987; 67: 514

3 Russel LJ, Aldrete JA. Intranasal ketamine: preliminary pharmacokinetics. In: Proceedings of the 9 th World Congress of Anesthesiologists. Washington DC, 1988: 864, Abstracts Vol

4 Walberg EJ, Wills RJ, Eckert J. Plasma concentrations of midazolam in children following intranasal administration. midazolam in children following

5 Avram MJ, Fragen RJ, Caldwell NJ. Dose finding and pharmacokinetic study of intramuscular midazolam. $₹$ Clin Pharmacol 1987; 27: 314.

\title{
Congenital third nerve palsy associated with mid-trimester amniocentesis
}

\author{
C K Patel, D S I Taylor, I M Russell-Eggitt, A Kriss, P Demaerel
}

Department of Ophthalmology, St George's Hospital Blackshaw Road, London SW17 00T C K Patel

Hospital for Sick Children, Great Ormond Street, London WC1N 1EH D S I Taylor I M Russell-Eggitt A Kriss

National Hospital for Nervous Diseases, Queen's Square, London WCIN 1EH

P Demaerel

Correspondence to: D S I Taylor.

Accepted for publication 19 March 1993
Amniocentesis is the presumed cause of many types of penetrating fetal injuries including skin marks, ${ }^{1}$ ocular perforation, ${ }^{2-7}$ limb, chest, and abdominal trauma. ${ }^{8-10}$ The following report describes how it may also cause neuro-ophthalmic disease and adds to other reports ${ }^{211}{ }^{12}$ that suggest it is an important cause of congenital intracranial damage.

\section{Case report}

A 3 month male infant was referred because his right eye had never opened. He was born at term following uneventful labour and vaginal delivery. Amniocentesis had been performed at 17 weeks of gestation because of advanced maternal age. Ultrasound was used to localise the placenta. Two unguided attempts were then made to aspirate amniotic fluid by stabs through one abdominal site. Both times the fluid was stained with blood, which to our knowledge was not analysed to determine its source.
Ocular examination showed a complete ptosis on the right. A diagnosis of total third nerve palsy was made by finding an externally ophthalmoplegic eye that was deviated down and out (Fig 1). Ocular movements on the left were normal. The infant fixated more actively with the

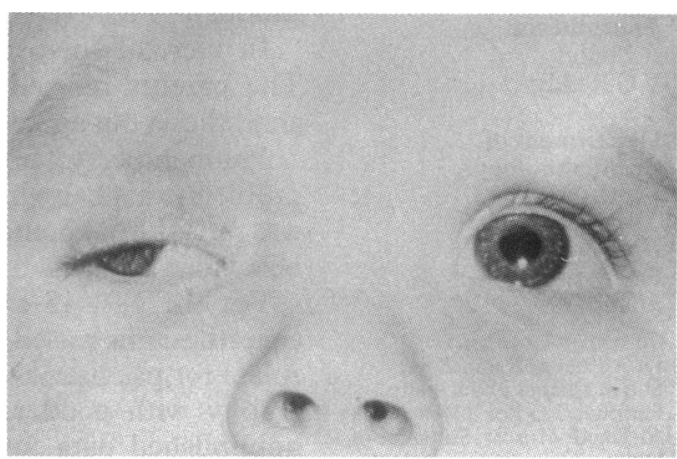

Figure 1 Right ptosis associated with hypotropia and exotropia. 
Figure 2 Flash VEPs and $E R G$ s from our patient (left upper trace) and agematched control (left lower trace) are shown on the left. $V E P s$ to pattern reversal using 100 second checks are shown on the right. VEPs are recorded from left occipital (L Occ), midoccipital (MOcc), and right occipital (R Occ) locations referred to a mid-frontal reference $(M F)$. The right eye $V E P$ s are better defined compared with the left eye's confirming the clinical suspicion that visual acuity was better in the paretic eye. The marked occipital asymmetry in the patient's $V E P$ s suggests right posterior hemisphere dysfunction (see text for fuller explanation).

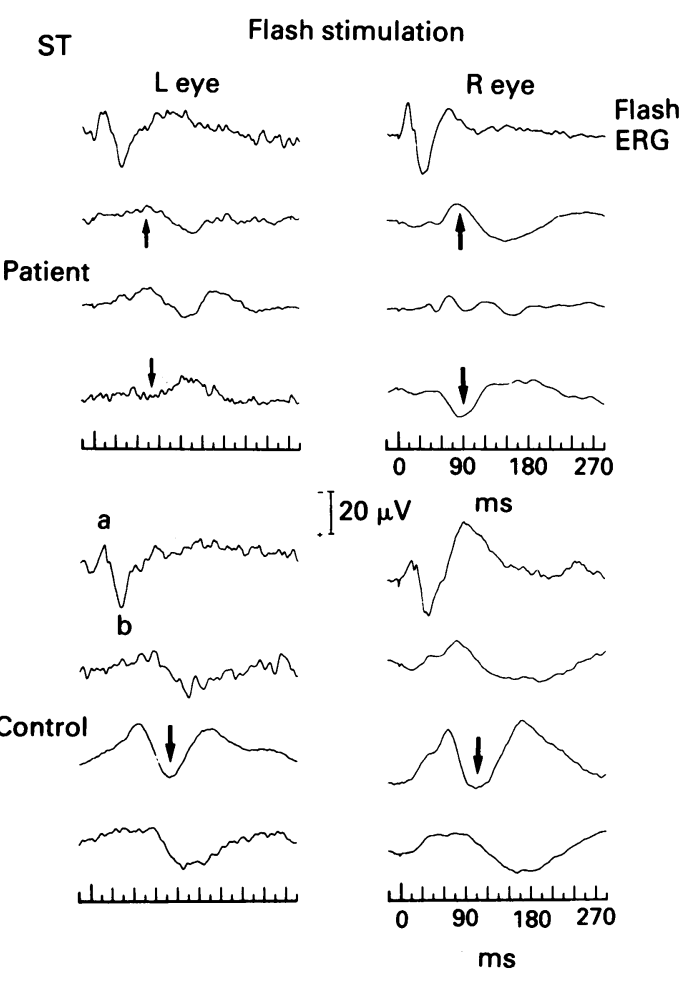

right eyelid raised suggesting that there was better vision in the paretic eye. There was no relative afferent pupil defect. Cycloplegic retinoscopy was +3.5 DS right and +4.0 DS left. The optic discs looked normal. Visual evoked potentials (VEPs) to flash and to pattern stimulation demonstrated left optic nerve dysfunction (Fig 2).

Physical examination showed a skin mark on the right temple (Fig 3). There was an area of pigmentation separating two linear scars which looked like puncture marks. There were no focal

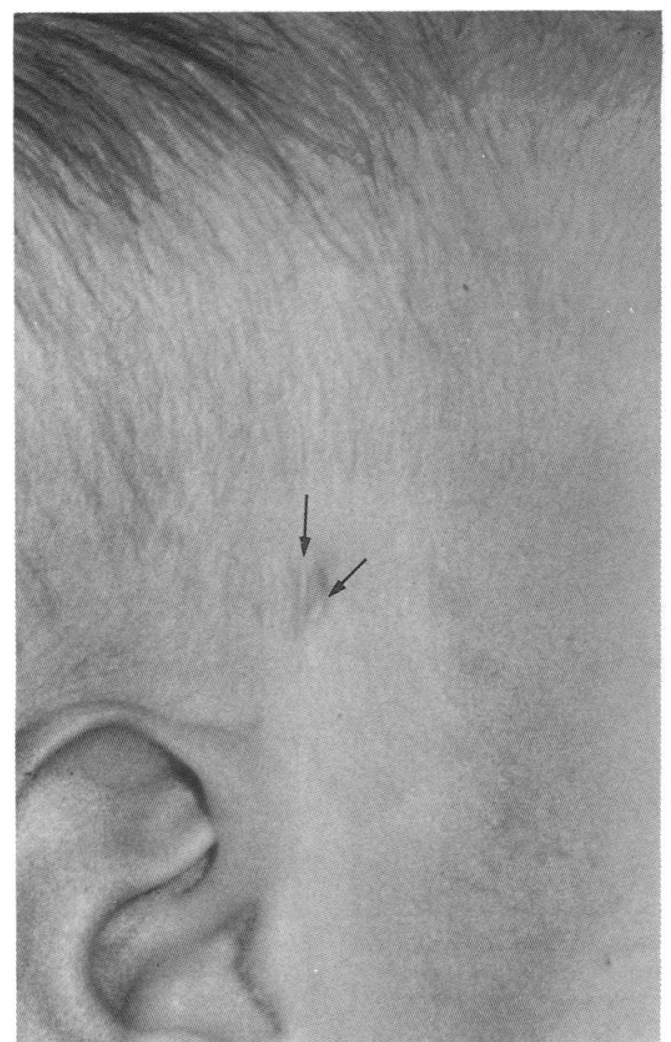

Pattern stimulation ST
Figure 3 Skin mark on the right temple. Note the pigmented area separating the two scars shown by the arrows. neurological limb signs. Magnetic resonance imaging showed right hemiatrophy of the cerebrum, cerebellum, and brain stem (Figs 4 and 5) with hypoplasia of the right middle cerebral circulation (Figs 4(a) and 6).

\section{Comment}

Mid-trimester amniocentesis has been linked with cerebral malformations in three previous cases. Naylor et $a l^{2}$ described an infant with convulsions, a gaze palsy, and a hemiparesis. They detected clinically a homonymous hemianopia in the absence of any consistent asymmetry in the VEPs. Computed tomography, however, showed a cerebral atrophy on the right. Youroukos et $a l^{12}$ have shown a porencephalic cyst in an infant who presented with vertical jerky eye movements, 'a probable left visual field defect', and a left hemiparesis. Chong et al ${ }^{11}$ reported a neonate with congenital hydrocephalus and a large subarachnoid cyst.

Ultrasound guidance was not used during amniocentesis for these cases although it did predetermine the location of the placenta in the cases described by Naylor and Chong. The use of real time scanning is likely to reduce the risk of inadvertent fetal puncture ${ }^{13}$ but does not necessarily eliminate it. $^{2}$ Blood staining of the amniotic fluid was a common feature of all three cases. Naylor et al proved fetal vascular disruption by demonstrating that the blood was $100 \%$ fetal. When an injury is suspected retrospectively this proof may not be available. A study has, however, shown that fetal blood is usually present when amniotic fluid is grossly stained suggesting that fetal injury may be common." Routine analysis of blood stained amniotic fluid by obstetricians would be valuable. Presumed cerebral puncture can be proved biochemically by examining amniotic fluid for the neural isoenzyme of acetylcholinesterase. ${ }^{2}$ 
Figure 4 Axial T2 weighted images showing atrophy of the right cerebral hemisphere and brainstem $(a, A)$. The temporal lobe is more severely affected with the formation of a

porencephalic cyst which communicates with the temporal horn of the right lateral ventricle $(a, B)$. The right internal carotid artery is hypoplastic $(a, C)$. There is a marked delay in cerebral myelination on the side of the paretic deviated eye compared with the normal left side (arrow, $b$ ).

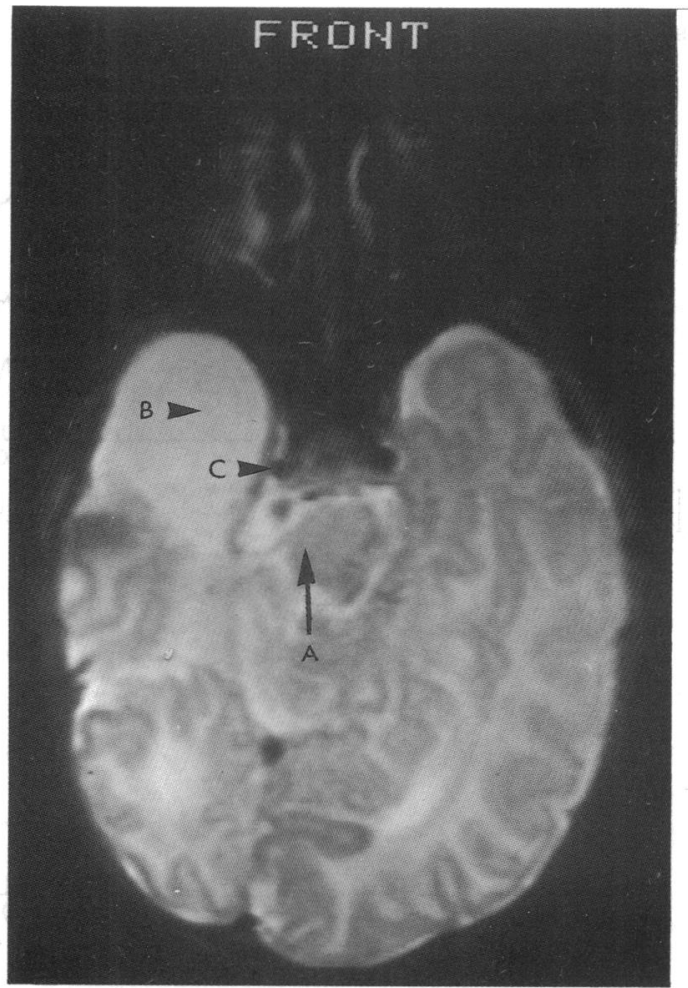

Fig $4 a$

Skin marks, compatible with needle injury, are a more permanent feature of injury during amniocentesis. In the case described by Chong et al a scar was serendipitously discovered after the infant's head was shaved in preparation for the insertion of a cerebrospinal fluid shunt. Such marks can be inconspicuous if fetal skin heals

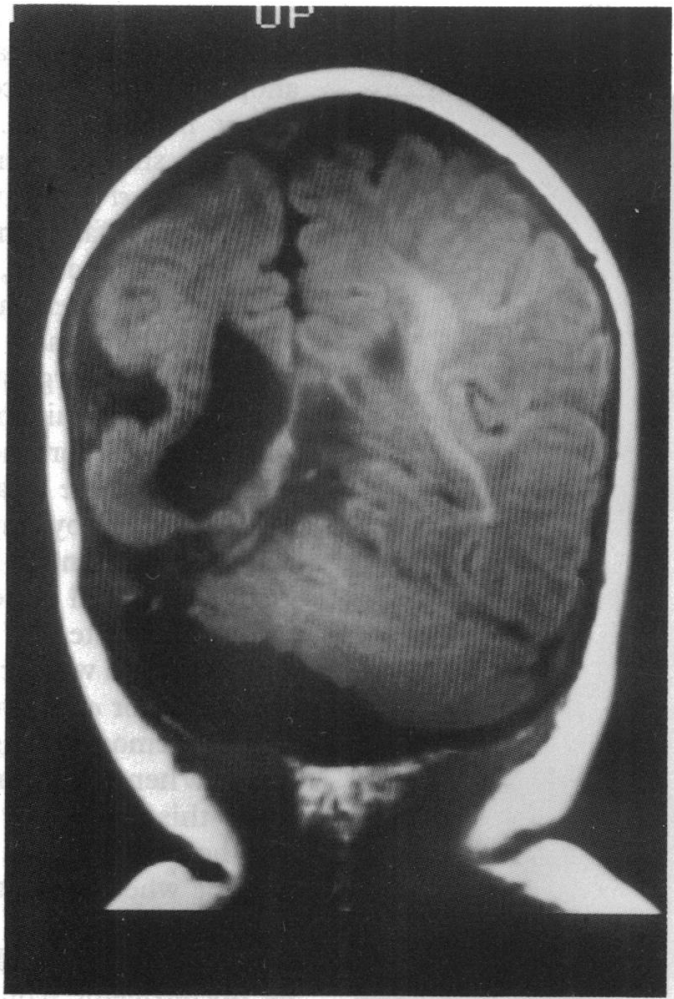

Figure 5 Coronal T1 weighted sequence showing atrophy of the right cerebral and cerebellar hemisphere. There is enlargement of the right lateral ventricle and widening of the subarachnoid space.

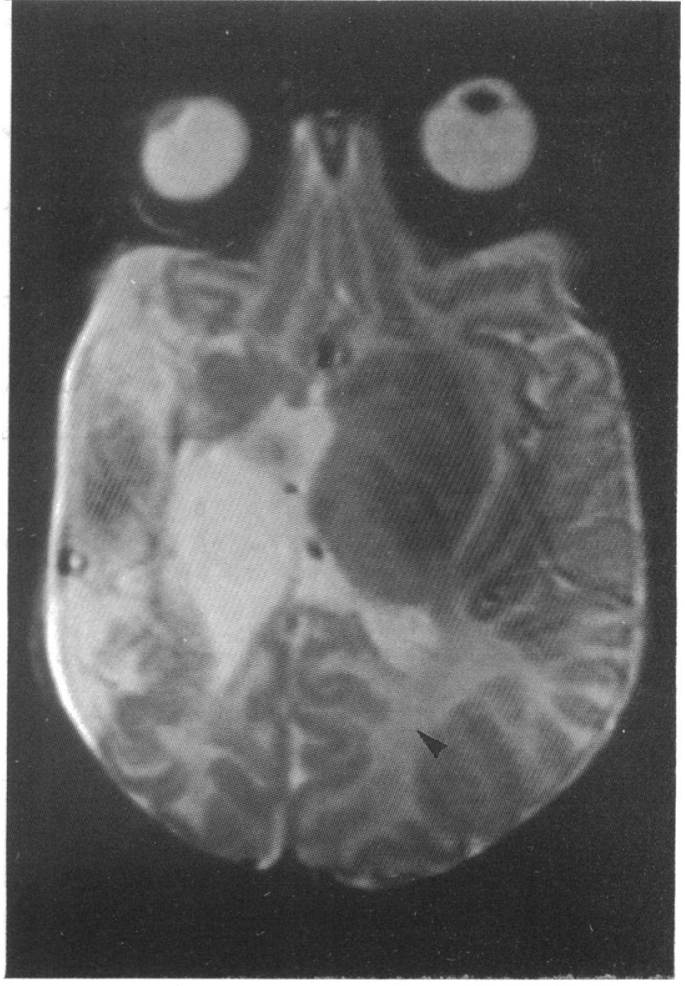

Fig $4 b$

rapidly and there is delayed traction on the epidermis caused by fibrosis. ${ }^{29}$ There should be a thorough examination of the head in all neonates where iatrogenic trauma is suspected even with recourse to shaving the head if the need for diagnosis is sufficiently great. Skin marks are usually dimpled or linear, ${ }^{8}$ but may be nodular

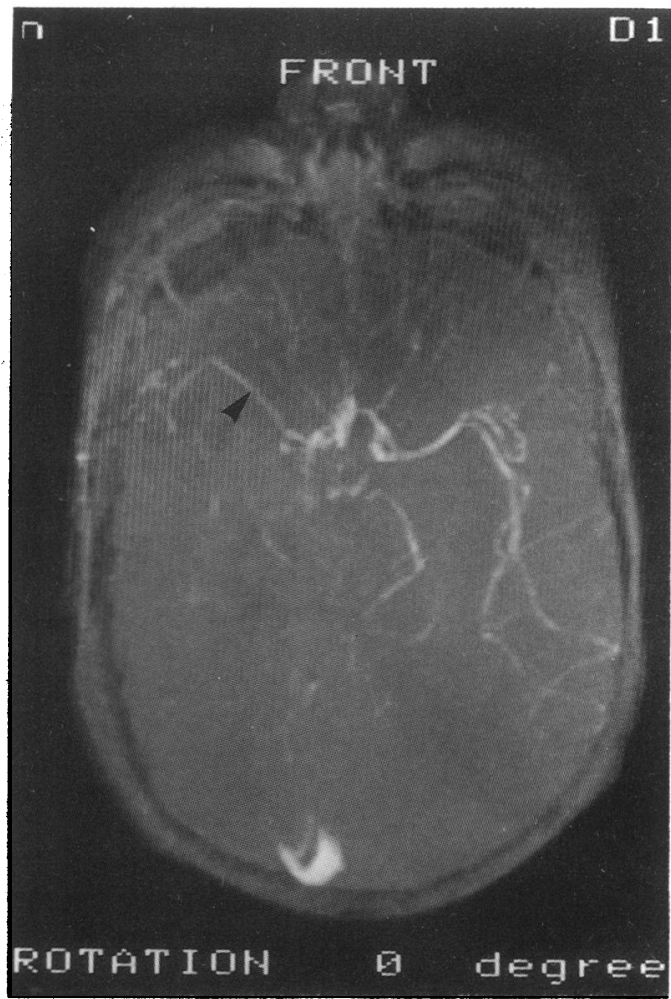

Figure 6 Magnetic resonance angiogram. The right middle cerebral artery appears hypoplastic with the left (arrow). There are a diminished number of cortical branches seen on the right side. 
and associated with a discharging sinus. ${ }^{12}$ The morphology of the pigmented lesion in our patient, the use of a single abdominal site during amniocentesis, and the small size of a 17 week fetal head makes it possible that the head was punctured twice in close proximity. An oblique rather than perpendicular penetrating injury is suggested by the presence of linear scars rather than dimples and could account for the extensive intracranial damage that occurred.

Congenital third nerve palsy is important to recognise and investigate because the neurodevelopmental prognosis is not as $\operatorname{good}^{1516}$ as previously thought. ${ }^{17} 18$ Trauma during vaginal birth or forceps delivery may damage the peripheral nerve. ${ }^{19}$ Necropsy, computed tomography, and magnetic resonance imaging have highlighted thromboembolic vascular disease as a cause of nuclear palsies. ${ }^{1520}$ Congenital heart disease and infective endocarditis are the most important causes of embolic disease in childhood. ${ }^{20}$ Mid-brain ischaemia has been reported following vertebral artery trauma during birth. ${ }^{21}$ The occurrence of pulmonary emboli following umbilical vein catheterisation in neonates may be relevant to our case. ${ }^{22}$ If the fetal venous circulation is traumatised during amniocentesis, emboli could be diverted into the systemic fetal circulation by the high pulmonary resistance. This could explain the involvement of the internal carotid and vertebrobasilar territories in our patient. The unilateral distribution of cerebral atrophy, however, favours direct trauma to the anterior and posterior cranial fossae. Magnetic resonance imaging supports this hypothesis by showing accentuated atrophy in the temporal lobe under the skin marks that were observed.

Previous reports have highlighted the finding of better vision in the paretic eye of patients with congenital third nerve palsy. ${ }^{1523}$ Where refractive errors or visual pathway defects are clinically inapparent it has been suggested that nystagmus may lead to amblyopia because of the fixational advantage conferred on the paretic eye. ${ }^{15}$ Nystagmus was absent in our patient but VEP testing showed its value by identifying subclinical optic nerve dysfunction to account for the reduced vision in the left eye. Figure 2 shows the occipital asymmetry in the VEPs to flash and pattern reversal stimulation. The main positivity of the pattern reversal VEP shows a paradoxical lateralisation as it is mainly distributed ipsilateral to the half field stimulated. Electrodes over the ipsilateral scalp are well placed for picking up activity generated at the posterior medial aspects of the activated hemisphere. We are surprised that asymmetry to pattern reversal was not detected in the patient described by Naylor ${ }^{2}$ but technical detail was not given.

We thank S C Patel and A Tank for their secretarial help.

1 Broome DP, Wilson MG, Weiss B, Kellog B. Needle puncture of fetus: a complication of second trimester amniocentesis. Am F Obstet Gynecol 1976; 126: 247-52.

2 Naylor G, Roper JP, Willshaw HE. Ophthalmic complications of amniocentesis. Eye 1990; 4: 845-9.

3 Cross HE, Maumenee AE. Ocular trauma during amniocentesis. Arch Ophthalmol 1973; 90: 303-4.

4 Merin S, Beyth Y. Uniocular congenital blindness as a complication of mid-trimester amniocentesis. Am $\mathrm{f}$ Ophthalmol 1989; 89: 299-301.

5 Fortin JG, Lemire J. Une complication oculaire de l'amniocentese. Can $\mathcal{F}$ Ophthalmol 1975; 10: 511-3.

6 Isenberg SJ, Heckenlively JR. Traumatised eye with retina damage from amniocentesis. $\mathcal{f}$ Pediatr Ophthalmo Strabismus 1985; 22: 65-7.

7 Admoni MM, BenEzra D. Ocular trauma following amniocentesis as the cause of leukocoria. $\mathcal{F}$ Pediatr Ophthalmol Strabismus 1988; 25: 196-7.

8 Creasman WT, Lawrence RA, Thiede HA. Fetal complications of amniocentesis. $¥ A M A$ 1968; 204: 949-52.

9 Epley SL, Hanson JW, Cruikshank DP. Fetal injury with midtrimester diagnostic amniocentesis. Obstet Gynecol 1979; 53: 77-80.

10 Egley CC. Laceration of fetal spleen during amniocentesis. Am f Obstet Gynecol 1973; 116: 582-3.

11 Chong SKF, Levitt GA, Lawson J, Lloyd U, Newman CGH Subarachnoid cyst with hydrocephalus - a complication of mid-trimester amniocentesis. Prenatal Diagnosis 1989; 9 : 677-9.

12 Youroukos S, Papdelis F, Matsanoitis N. Porencephalic cyst after amniocentesis. Arch Dis Child 1980; 55: 814-5.

13 Williamson RA, Varner MW, Grant SS. Reduction in amniocentesis risks using a real time needle guide procedure. Obstet Gynecol 1985; 65: 751-5.

14 Young PE, Matson MR, Jones OW. Fetal exsanguination and other vascular injuries from mid-trimester genetic amnioother vascular injuries from mid-trimester ge

15 Hamed LM. Associated neurological and ophthalmologic findings in congenital oculomotor nerve palsy. Ophthalmology 1991; 98: 708-14.

16 Balkan R, Hoyt CS. Associated neurological abnormalities in congenital 3rd nerve palsy. Am $\mathcal{f}$ Ophthalmol 1984; 97 315-9.

17 Victor DI. The diagnosis of congenital unilateral 3rd nerve palsy. Brain 1976; 99: 711-8.

18 Miller NR. Solitary oculomotor nerve palsy in childhood. Am F Ophthalmol 1977; 83: 106-11.

19 Miller NR. Walsh $\mathcal{E}$ Hoyt's clinical neuro-ophthalmology. 4th Ed. Baltimore: William \& Wilkins, 1985: Vol 2.652-4.

20 Norman MG. Unilateral encephalomalacia in cranial nerve nuclei in neonates: report of two cases. Neurology 1974; 24 : nuclei in

21 Yates PO. Birth trauma to the vertebral arteries. Arch Dis Child 1959; 34: 436-41.

22 Joachim Wigger H, Bransilver BR, Blanc WA. Thromboses due to catheterization in infants and children. $\mathcal{F}$ Pediat 1970; 76: 1-11.

23 Keith CG. Congenital ocular palsy. Br f Ophthalmol 1972; 56 356-61. 